\title{
Increased Glycogen Synthase Kinase-3 $\beta$ mRNA Level in the Hippocampus of Patients with Major Depression: A Study Using the Stanley Neuropathology Consortium Integrative Database
}

\author{
Dong Hoon Oh, Yong Chon Park and Seok Hyeon Kim ${ }^{\bowtie}$ \\ Department of Neuropsychiatry, College of Medicine and Institute of Mental Health, Hanyang University, Seoul, Korea
}

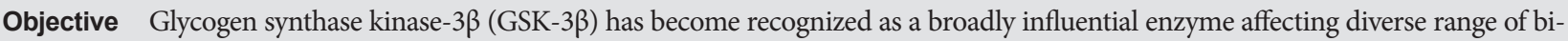
ological functions, including gene expression, cellular architecture, and apoptosis. The results of previous studies suggest that GSK- $3 \beta$ activity may be increased in the brain of patients with major depressive disorders (MDD). A recent animal study reported increased GSK$3 \beta$ messenger ribonucleic acid (mRNA) level in the hippocampus of those with depression. However, few studies have investigated GSK$3 \beta$ activity in the brain of patients with MDD.

Methods In order to test whether patients with MDD have an increase in GSK-3 $\beta$ activity in the brain compared to normal controls, we explored GSK-3 $\beta$ expression level in all brain regions by using the Stanley Neuropathology Consortium Integrative Database (SNCID), which is a web-based method of integrating the Stanley Medical Research Institute data sets.

Results The level of GSK- $3 \beta$ mRNA expression in the hippocampus was significantly increased in the MDD group ( $n=8$ ) compared with the control group $(n=12, p<0.05)$. Spearman's test also reveals that GSK-3 $\beta$ mRNA expression levels were significantly correlated with nitric oxide synthase $1(\mathrm{NOS} 1)(\rho=0.70, \mathrm{p}<0.0001)$ and stathmin-like $3(\operatorname{STMN} 3)(\rho=0.70, \mathrm{p}<0.0001)$ in the hippocampus.

Conclusion Our results correspond with the results of previous animal studies that reported increased GSK-3 $\beta$ activity in the hippocampus of those with depression. Our findings also suggest that oxidative stress-induced neuronal cell death and abnormal synaptic plasticity in the hippocampus may play important roles in the pathophysiology of major depression.

Psychiatry Investig 2010;7:202-207
\end{abstract}

Key Words Data mining, Glycogen synthase kinase-3 $\beta$, Hippocampus, Major depressive disorder.

\section{INTRODUCTION}

Glycogen synthase kinase-3 (GSK-3) was first identified as a protein kinase that phosphorylates and inhibits glycogen synthase. ${ }^{1}$ GSK-3 exists in two closely related isoforms, GSK-3a and GSK-3 $\beta$. GSK-3 $\beta$ is an important regulatory protein involved in many intracellular signaling pathways leading to neural plasticity adaptations and influencing cell survival. ${ }^{2}$ Overexpression of GSK-3 $\beta$ in neurons increased neuronal apoptosis. ${ }^{3}$

Received: March 29, 2010 Revised: May 27, 2010

Accepted: June 17, 2010 Available online: July 12, 2010

$\triangle$ Correspondence: Seok Hyeon Kim, MD, PhD

Department of Neuropsychiatry, College of Medicine and Institute of Mental Health, Hanyang University, Haengdang 1-dong, Seongdong-gu, Seoul 133-792, Korea

Tel: +82-2-2290-8426, Fax: +82-2-2298-2055

E-mail: shkim1219@hanyang.ac.kr

(a) This is an Open Access article distributed under the terms of the Creative Commons Attribution Non-Commercial License (http://creativecommons.org/licenses/by$\mathrm{nc} / 3.0$ ) which permits unrestricted non-commercial use, distribution, and reproduction in any medium, provided the original work is properly cited.
In psychiatry, GSK-3 $\beta$ has been highlighted by the discovery of its inhibition by lithium, one of the most effective drugs for the treatment of bipolar disorder. ${ }^{4}$ Recently, two animal studies in 2004 demonstrated that rats treated with GSK-3 inhibitors showed reduced duration of immobility when exposed to the forced swim test and suggested the potential of GSK-3 inhibitors as antidepressants. ${ }^{5,6} \mathrm{GSK}-3 \beta$ activity would be also associated with serotonergic regulation which is closely related with mood disorder. Stimulation of 5HT1A receptors caused phosphorylation (inactivation) of GSK-3 $\beta$, whereas 5HT2 receptor stimulation mediated dephosphorylation (activation) of GSK-3 $3 .^{7}$

Several previous studies suggest that GSK- $3 \beta$ may be associated with the pathophysiology of major depressive disorders (MDD). A human post-mortem study indicated that GSK-3 $\beta$ activity of the ventral prefrontal cortex was increased in patients with major depression compared with control subjects. ${ }^{8}$ A genetic study showed GSK- $3 \beta$ genetic variants play a role in 
the antidepressant therapeutic response. ${ }^{9}$ Reduced gray matter volume in the right hippocampus and bilateral superior temporal gyrus is related with a common intronic polymorphism (rs6438552) at the GSK-3 $\beta$ locus in patients with MDD. ${ }^{10}$

In animal studies, mild traumatic brain injury (mTBI) resulted in depressive behavior and increased phosphorylation of inhibitory site serine 9 of GSK-3 $\beta$ in the hippocampus of the rat. Pretreatment with GSK-3 inhibitors prevented mTBIinduced depression. ${ }^{11}$ A chronic mild stress model induced depressive-like behavior that was paralleled by hypercortisolemia and an increase of GSK-3 $\beta$ messenger ribonucleic acid (mRNA) expression in the hippocampus. ${ }^{12}$

However, there are few reports that have investigated the GSK-3 $\beta$ level in the brain of patients with MDD. We hypothesized that patients with MDD have an increased level of GSK$3 \beta$ in the brain compared to that of normal controls. In order to test this hypothesis, we explored a specific marker type (GSK$3 \beta$ ) in all brain regions by using the Stanley Neuropathology Consortium Integrative Database (SNCID)(http://sncid.stanleyresearch.org/) which is a web-based tool to integrate the Stanley Medical Research Institute (SMRI) data sets. We also attempted to identify which markers correlate with GSK-3 $\beta$ levels in a specific brain region.

\section{METHODS}

\section{Post-mortem brain tissues}

The SNC is a subset of 60 specimens from the collection, well-matched groups of 15 each with diagnoses of schizophrenia, bipolar disorder, MDD without psychotic features, and normal controls. Diagnoses were made independently by two senior psychiatrists according to DSM-IV. The four groups are matched by age, sex, race, postmortem interval (PMI), brain $\mathrm{pH}$, side of brain, and mRNA quality. Specimens are collected with the permission of the families in a standardized manner, with half of each specimen being frozen and half fixed in formalin.

More than 75,000 sections and blocks from the Consortium have been sent to over 50 research groups worldwide to carry out a wide variety of assessments. The details of the sample collection have been reported previously. ${ }^{13}$

\section{Stanley Neuropathology Consortium Integrative Database}

The SNCID has been generated with multiple datasets from research groups located all over the world. Research is still being conducted with SNC tissue, and data sets are continually being returned to the SMRI. Hence, this SNCID will continue to be updated. The SNCID is freely available to all users. However, for commercial use, agreement and permission should be obtained from the SMRI. Descriptive statistical and variance analysis modules (R-scripts) enable users to identify neuropathological traits in multiple brain areas for psychiatric disorders such as bipolar disorder, depression and schizophrenia.

\section{Statistical analysis}

The effect of the disorders on the neuropathology markers (such as GSK-3 $\beta$ ) was initially tested by variance analysis. The nonparametric Kruskal-Wallis test was used because not all of the traits are normally distributed. If there was a significant effect of the disorders on a trait $(\mathrm{p}<0.05)$, a post hoc Mann-Whitney U-test for group differences between each disorder group and unaffected controls was used (R-packages, http://www.rproject.org/). In order to determine which markers correlate with GSK-3 $\beta$ in specific brain regions, the Spearman's rank correlation test was performed. ${ }^{14}$

To examine whether the neuropathology marker was affected by age, brain $\mathrm{pH}$, duration of illness, lifetime exposure to antipsychotics and PMI, we determined their interrelationships by Spearman's correlation analysis. The effect of categorical variables (such as sex, smoking, suicide, psychotic feature, alcohol, and drug abuse) on the neuropathology markers was also tested by nonparametric variance analysis. p-values less than 0.05 were considered significant. Using analysis of covariance (ANCOVA) or partial correlation analysis, the effect of the neuropathology marker is estimated after controlling for the effect of confounding variables.

For this study, we also downloaded the raw data from the SNCID website (repository database) for confirming online statistical results and further statistical analysis by using the Statistical Package for the Social Sciences (SPSS) 17.0 software (SPSS Inc., Chicago, IL, USA).

\section{RESULTS}

In the SNCID, we found 3 studies which investigated GSK$3 \beta$ expression in the brain regions (Table 1$)$. Among them, when we confirmed the results of variance analysis in all three studies, we found William Deakin's study showed statistically significant results. Namely, GSK-3 $\beta$ mRNA expression in the hippocampus only showed significant findings in the variance analysis.

\section{The variance analysis of GSK- $3 \beta$ mRNA expression in the hippocampus}

The results of the variance analysis are summarized in Table 2. The level of GSK-3 $\beta$ mRNA expression in the hippocampus was significantly increased in the MDD group $(n=8,0.014 \pm$ $0.004)$ compared with the unaffected controls $(n=12,0.010 \pm$ 0.003) $(\mathrm{p}<0.05)$ (Fig. 1)(Table 3). 
Increased GSK-3 $\beta$ Level in the Hippocampus of MDD

Table 1. Result of exploring GSK-3 $\beta$ in all brain regions by using SNCID

\begin{tabular}{cllll}
\hline Marker & \multicolumn{1}{c}{ Researcher } & Brain/Region & Experimental method & Published \\
\hline GSK-3 $\beta$ & Belmaker, Robert & Frontal & Western blot & Yes \\
GSK-3 $\beta$ & Yoshikawa, Takeo & Frontal (BA6) & Western blot & No \\
GSK-3 $\beta^{*}$ & Deakin, William & Hippocampus & qRT-PCR & No \\
\hline
\end{tabular}

*data normalized to geometric mean of succinate dehydrogenase complex subunit A (SDHA) and beta actin (ACTB) amounts. GSK-3 $\beta$ : glycogen synthase kinase-3 $\beta$, SNCID: Stanley Neuropathology Consortium Integrative Database, qRT-PCR: quantitative reverse transcriptase polymerase chain reaction, BA: Brodmann area

Table 2. Result of variance analysis of GSK-3ß mRNA expression from hippocampus

\begin{tabular}{|c|c|c|c|c|c|c|c|c|c|}
\hline \multirow{2}{*}{ Marker } & \multirow{2}{*}{ Brain region } & \multicolumn{2}{|c|}{ BP, MDD, Schiz, control } & \multicolumn{2}{|c|}{ BP vs. control } & \multicolumn{2}{|c|}{ MDD vs. control } & \multicolumn{2}{|c|}{ Schiz vs. control } \\
\hline & & $\mathrm{H}$ & $\mathrm{p}$ & Subjects & $\mathrm{p}$ & Subjects & $\mathrm{p}$ & Subjects & $\mathrm{p}$ \\
\hline GSK-3 $\beta$ mRNA* & Hippocampus & 7.883 & 0.048 & $11: 12$ & 0.622 & $8: 12$ & 0.025 & $11: 12$ & 0.441 \\
\hline
\end{tabular}

Statistical significance test was done by Kruskal-Wallis test or Mann-Whitney U-test. *data normalized to geometric mean SDHA and ACTB

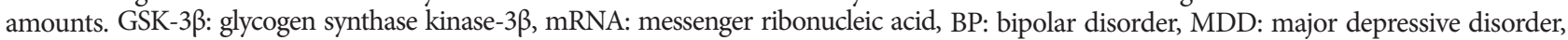
Schiz: schizophrenia

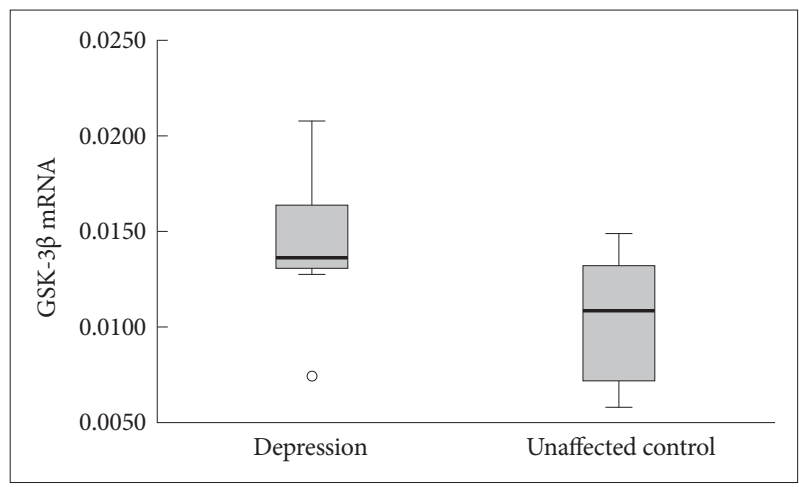

Figure 1. Comparison of GSK-3ß mRNA expression level in depression group and control group (data normalized to geometric mean of SDHA and ACTB amounts). GSK-3ß: glycogen synthase kinase-3 $\beta$, mRNA: mesenger ribonucleic acid.

\section{Correlation analysis}

Spearman's rank correlation test reveals that two markers in the hippocampus were significantly correlated $(\mathrm{p}<0.0001)$ with GSK-3 $\beta$ mRNA levels in the hippocampus (Figures 2 and 3). GSK-3 $\beta$ mRNA expression levels were significantly correlated with nitric oxide synthase $1(\mathrm{NOS} 1)(\rho=0.70, \mathrm{p}<0.0001)$ and stathmin-like 3 (STMN3) $(\rho=0.70, \mathrm{p}<0.0001)$ in the hippocampus (Table 4). In the results of correlation analysis from 20 MDD and unaffected control subjects, a similar correlation pattern was also found between GSK-3 $\beta$, NOS1 and STMN3. However, the result was statistically significant only between GSK-3 $\beta$ and STMN3 (Table 5).

\section{Effect of confounding variables}

We could not find any significant association between GSK$3 \beta$ mRNA expression in the hippocampus and confounding variables (11 descriptive variables mentioned in the method part). However, we observed a weak correlation between GSK$3 \beta$ mRNA levels and PMI in the hippocampus $(\rho=-0.30, p=$ 0.0522). On the basis of ANCOVA with adjustment for age and
Table 3. Demographic and clinical characteristics of study subjects

\begin{tabular}{lcc}
\hline & MDD & Unaffected controls \\
\hline Age (year) & $44.25 \pm 10.98$ & $47.33 \pm 11.33$ \\
Sex & $4 \mathrm{~F}, 4 \mathrm{M}$ & $6 \mathrm{~F}, 6 \mathrm{M}$ \\
Race & $8 \mathrm{~W}$ & $11 \mathrm{~W}, 1 \mathrm{NW}$ \\
Duration of illness & $10.56 \pm 8.38$ & 0 \\
Suicide & 4 & 0 \\
PMI (hour) & $31.62 \pm 9.73$ & $23.41 \pm 11.14$ \\
Brain pH & $6.16 \pm 0.18$ & $6.23 \pm 0.25$ \\
\hline
\end{tabular}

MDD: major depressive disorder, F: female, M: male, W: white, NW: non-white, PMI: postmortem interval

PMI, a more marked statistical difference in GSK-3 $\beta$ mRNA level was identified between the MDD group and the control group, as $\mathrm{p}$ value of 0.003 .

In order to statistically control for the general effects of age, sex, clinical diagnosis, brain $\mathrm{pH}$ and $\mathrm{PMI}$, a partial correlation analysis was also performed. We also acquired significant results in the partial correlation analysis between GSK-3 $\beta$, NOS1 and STMN3 (Table 5).

\section{DISCUSSION}

The first objective of this study was achieved by identifying increased GSK- $3 \beta$ mRNA expression in the hippocampus in the MDD group as compared to the control group. This result corresponds with the results of previous studies which have suggested that GSK-3 $\beta$ activity (or level) is increased significantly in the brain of patients with MDD. ${ }^{5,6,8,9,11,12}$ This study indicated the hippocampus as the major region that is associated with increased GSK-3 $\beta$ activity in MDD among various brain regions.

The hippocampus has been known to be involved in the pathophysiology of MDD. A meta-analysis of 12 studies of 

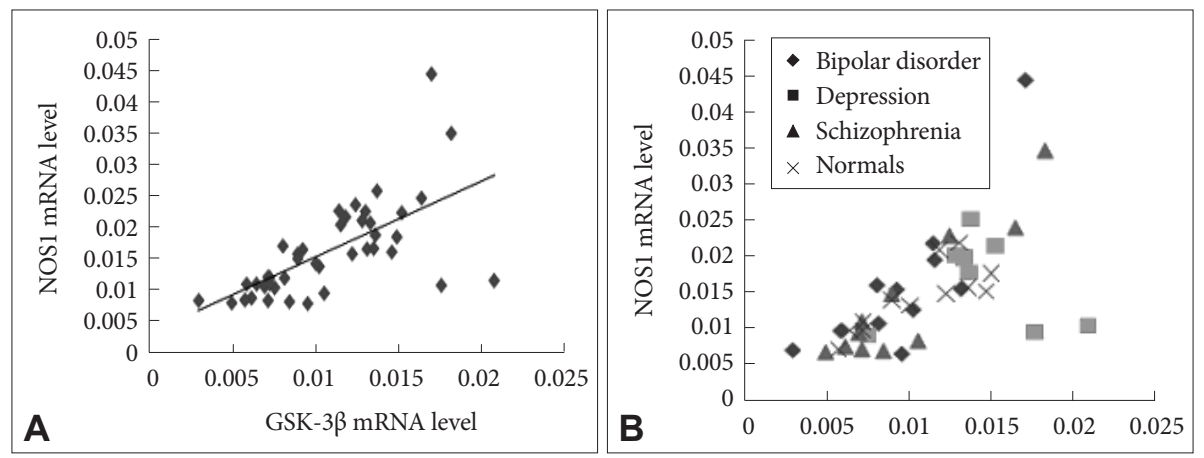

Figure 2. Correlation between hippocampal GSK-3ß \& NOS1 level in all the subjects $(N=42)$ (data normalized to geometric mean of SDHA and ACTB amounts). A: The expression level of GSK-3 $\beta$ mRNA was positively correlated with NOS1 mRNA expression level, and the Spearman's

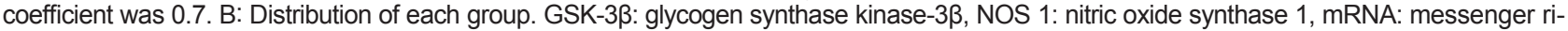
bonucleic acid.
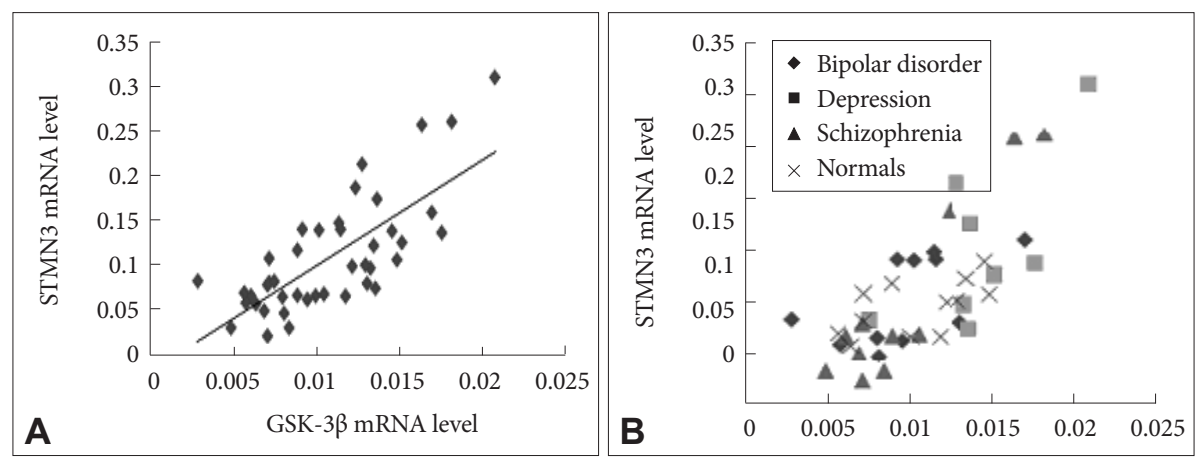

Figure 3. Correlation between hippocampal GSK-3 $\beta$ \& STMN3 level in all the subjects $(\mathrm{N}=42)$ (data normalized to geometric mean of SDHA and ACTB amounts). A: The expression level of GSK-3 $\beta$ mRNA was positively correlated with the STMN3 mRNA expression level, and the Spearman's

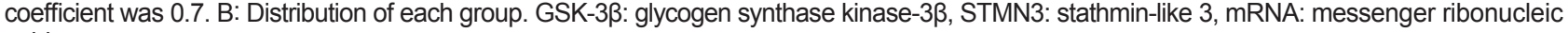
acid.

Table 4. The results of correlation analysis

\begin{tabular}{llccc}
\hline \multicolumn{1}{c}{ Marker } & Brain/Region & Method & $\rho$ & p-value \\
\hline GSK-3 $\beta^{*}$ & Hippocampus & qRT-PCR & 1.000 & 0.000 \\
NOS1 $^{*}$ & Hippocampus & qRT-PCR & 0.700 & 0.000 \\
STMN3 $^{*}$ & Hippocampus & qRT-PCR & 0.700 & 0.000 \\
\hline
\end{tabular}

*data normalized to geometric mean of SDHA and ACTB amounts. GSK-3 $\beta$ : glycogen synthase kinase- $3 \beta$, NOS 1: nitric oxide synthase 1 , STMN3: stathmin-like 3, qRT-PCR: quantitative reverse transcriptase polymerase chain reaction, $\rho$ : spearman rho correlation coefficient

MDD reported that hippocampal volume is reduced in patients with MDD, perhaps as a consequence of repeated periods of MDD. ${ }^{15}$ However, the mechanism of the hippocampal volume reduction is still unknown. Based on our results and the report of Inkster et al. ${ }^{10}$ we cautiously suggest that the elevated GSK-3 $\beta$ level-inflicted neuronal cell death pathways may be associated with decreased hippocampal volume.

Although the mechanism by which overexpressed GSK-3 $\beta$ causes apoptosis has not been fully identified, GSK3 has been known to promote the intrinsic apoptotic signaling pathway by regulating transcription factors that control the expression of pro-apoptotic proteins (e.g., Bax, Bim) and anti-apoptotic proteins (e.g., Bcl-2, Mcl-1, Bcl-xL), by promoting microtubule disruption and cell structural changes that occur during apoptosis, and by promoting disruption of mitochondria (e.g., cytochrome c).$^{16}$ As such, we have also attempted to find experimental data related to apoptotic signaling pathway associated with increased GSK-3 $\beta$ expression in the hippocampus by using the SNCID. However, as we were unable to find relevant data, we could not proceed with further analysis.

Our results also highlight correlations between GSK-3 $\beta$, NOS1 and STMN3 in the hippocampus. NOS1 produces NO in nervous tissue in both the central and peripheral nervous system. ${ }^{17}$ Physiological amounts of NO are neuroprotective. If it is produced in excess, or if a cell is under conditions of oxidative stress, NO becomes noxious and may undergo an oxidation-reduction reaction to form toxic compounds known as reactive nitrogen species (RNS), which cause cell damage. Both NO and RNS have a pathogenetic role in the onset and development of neurodegenerative disorders. ${ }^{18} \mathrm{~A}$ recent study found that hippocampal NOS1 over-expression and impaired neurogenesis were observed in the mice exposed to chronic mild stress (CMS) that exhibited behavioral changes typical of depression. The CMS-induced behavioral despair and hippocam- 
Increased GSK-3 $\beta$ Level in the Hippocampus of MDD

Table 5. Correlation between expression levels of GSK-3ß mRNA and NOS1 mRNA and STMN3 in the hippocampus

\begin{tabular}{|c|c|c|c|c|c|}
\hline \multirow{2}{*}{ Markers } & \multirow{2}{*}{ Subjects } & \multicolumn{2}{|c|}{ Spearman's correlation } & \multicolumn{2}{|c|}{ Partial correlation } \\
\hline & & Coefficient & p-value & Coefficient & p-value \\
\hline \multirow[t]{2}{*}{ NOS1 mRNA* } & Total $(\mathrm{N}=42)$ & 0.700 & 0.000 & 0.595 & 0.000 \\
\hline & MDD+Controls $(\mathrm{N}=20)$ & 0.437 & 0.054 & - & - \\
\hline \multirow[t]{2}{*}{ STMN3 mRNA* } & Total $(\mathrm{N}=42)$ & 0.700 & 0.000 & 0.728 & 0.000 \\
\hline & MDD+Controls $(\mathrm{N}=20)$ & 0.671 & 0.001 & - & - \\
\hline
\end{tabular}

*data normalized to geometric mean of SDHA and ACTB amounts. GSK-3 $\beta$ : glycogen synthase kinase-3 $\beta$, mRNA: mesenger ribonucleic acid, NOS: nitric oxide synthase 1, STMN3: stathmin-like 3, MDD: major depressive disorder

pal neurogenesis impairment were prevented and reversed in the null mutant mice lacking the NOS1 gene (NOS1-/-) and in the mice receiving NOS1 inhibitor. ${ }^{19}$ Hippocampal NOS1 expression was also increased in the CA1 region of patients with major depression. This was significantly more marked in samples from the right hemisphere. ${ }^{20}$

STMN3 belongs to the stathmin family. Stathmin is a member of a novel class of microtubule-destabilizing proteins that regulate the dynamics of microtubule polymerization and depolymerization. ${ }^{21}$ Stathmin is highly enriched in the lateral nucleus of amygdala, the main input of fearful information about the unconditioned stimuli and conditioned stimuli to the amygdala and the site of their convergence during fear learning. Amygdala-enriched stathmin is required for the expression of innate fear and the formation of memory for learned fear. ${ }^{22}$ The role of stathmin in the human hippocampus is still unknown to us. However, in our study, we also found that the STMN3 mRNA expression level was slightly increased in the hippocampus of patients with major depression compared to normal controls, but there was no statistical significance observed between the two groups ( $\mathrm{p}$-value 0.069). Based on the results of previous research, we cautiously speculate that stathmin is involved in modulating hippocampal synaptic plasticity in the pathogenic process of major depression.

In the previous research using postmortem brain tissue from SNC, the subjects with MDD had significantly lower hippocampal 5-HT1A mRNA expression compared with the normal control group. ${ }^{23}$ Decreased 5HT1A and increased GSK-3 $\beta$ expression in the hippocampus of patients with MDD would be associated with the hypothesis that low 5HT1A activation may result in deficient inhibitory control of GSK-3 $\beta$ through impaired phosphorylation in the MDD.

Although this study provides interesting points for further exploration and evaluation, we believe that it should be considered exploratory at this stage. Future work will be needed to explore the biological pathway associated with increased GSK$3 \beta$ level and its function in the hippocampus of patients with MDD. Our study has also several limitations, mostly stemming from small sample size of the original data and preliminary exploratory study design using an online web-based database.
In this study, we found significant correlation between GSK$3 \beta$ and other molecules. However, a correlation does not mean that one variable causes the other. In particular, the expression levels of many genes and some cytoarchitectural traits could be affected by confounding factors such as age, sex, brain $\mathrm{pH}$, PMI, drug and alcohol histories in studies using human postmortem brain tissue. ${ }^{24}$ A causal relationship between two variables should be cautiously interpreted.

Summing up, our results correspond with the results of previous animal study that reported increased GSK- $3 \beta$ mRNA expression in the hippocampus of those with depression. Our findings also suggest that oxidative stress-induced neuronal cell death, abnormal synaptic plasticity, and serotonergic dysregulation in the hippocampus may play important roles in the pathophysiology of major depression.

\section{Acknowledgments}

This research was supported by a grant of the Korea Health $21 \mathrm{R} \& \mathrm{D}$ project, Ministry of Health and Welfare, Republic of Korea (A050047). We would like to thank the SMRI collaborators who allow SNCID to be freely available to all users, and also all investigators generating original data in the SMRIDB, in particular Dr. William Deakin and Carla Toro.

\section{REFERENCES}

1. Embi N, Rylatt DB, Cohen P. Glycogen synthase kinase-3 from rabbit skeletal muscle. Separation from cyclic-AMP-dependent protein kinase and phosphorylase kinase. Eur J Biochem 1980;107:519-527.

2. Michelon L, Meira-Lima I, Cordeiro Q, Miguita K, Breen G, Collier D, et al. Association study of the INPP1, 5HTT, BDNF, AP-2beta and GSK3beta GENE variants and restrospectively scored response to lithium prophylaxis in bipolar disorder. Neurosci Lett 2006;403:288-293.

3. Hetman M, Cavanaugh JE, Kimelman D, Xia Z. Role of glycogen synthase kinase-3beta in neuronal apoptosis induced by trophic withdrawal. J Neurosci 2000;20:2567-2574.

4. Klein PS, Melton DA. A molecular mechanism for the effect of lithium on development. Proc Natl Acad Sci U S A 1996;93:8455-8459.

5. Gould TD, Einat H, Bhat R, Manji HK. AR-A014418, a selective GSK3 inhibitor, produces antidepressant-like effects in the forced swim test. Int J Neuropsychopharmacol 2004;7:387-390.

6. Kaidanovich-Beilin O, Milman A, Weizman A, Pick CG, Eldar-Finkelman H. Rapid antidepressive-like activity of specific glycogen synthase kinase-3 inhibitor and its effect on beta-catenin in mouse hippocampus. Biol Psychiatry 2004;55:781-784.

7. Li X, Zhu W, Roh MS, Friedman AB, Rosborough K, Jope RS. In vivo regulation of glycogen synthase kinase-3beta (GSK3beta) by serotonergic activity in mouse brain. Neuropsychopharmacology 2004;29: 
1426-1431.

8. Karege F, Perroud N, Burkhardt S, Schwald M, Ballmann E, La Harpe $\mathrm{R}$, et al. Alteration in kinase activity but not in protein levels of protein kinase B and glycogen synthase kinase-3beta in ventral prefrontal cortex of depressed suicide victims. Biol Psychiatry 2007;61:240-245.

9. Tsai SJ, Liou YJ, Hong CJ, Yu YW, Chen TJ. Glycogen synthase kinase3beta gene is associated with antidepressant treatment response in Chinese major depressive disorder. Pharmacogenomics J 2008;8:384-390.

10. Inkster B, Nichols TE, Saemann PG, Auer DP, Holsboer F, Muglia P, et al. Association of GSK3beta polymorphisms with brain structural changes in major depressive disorder. Arch Gen Psychiatry 2009;66:721-728.

11. Shapira M, Licht A, Milman A, Pick CG, Shohami E, Eldar-Finkelman H. Role of glycogen synthase kinase-3beta in early depressive behavior induced by mild traumatic brain injury. Mol Cell Neurosci 2007;34: 571-577.

12. Silva R, Mesquita AR, Bessa J, Sousa JC, Sotiropoulos I, Leão P, et al. Lithium blocks stress-induced changes in depressive-like behavior and hippocampal cell fate: the role of glycogen-synthase-kinase-3beta. Neuroscience 2008;152:656-669.

13. Torrey EF, Webster M, Knable M, Johnston N, Yolken RH. The stanley foundation brain collection and neuropathology consortium. Schizophr Res 2000;44:151-155.

14. Kim S, Webster MJ. Correlation analysis between genome-wide expression profiles and cytoarchitectural abnormalities in the prefrontal cortex of psychiatric disorders. Mol Psychiatry 2010;15:326-336.

15. Videbech P, Ravnkilde B. Hippocampal volume and depression: a meta-analysis of MRI studies. Am J Psychiatry 2004;161:1957-1966.
16. Beurel E, Jope RS. The paradoxical pro- and anti-apoptotic actions of GSK3 in the intrinsic and extrinsic apoptosis signaling pathways. Prog Neurobiol 2006;79:173-189.

17. Andoh T, Lee SY, Chiueh CC. Preconditioning regulation of bcl-2 and p66shc by human NOS1 enhances tolerance to oxidative stress. FASEB J 2000;14:2144-2146.

18. Calabrese V, Mancuso C, Calvani M, Rizzarelli E, Butterfield DA, Stella AM. Nitric oxide in the central nervous system: neuroprotection versus neurotoxicity. Nat Rev Neurosci 2007;8:766-775.

19. Zhou QG, Hu Y, Hua Y, Hu M, Luo CX, Han X, et al. Neuronal nitric oxide synthase contributes to chronic stress-induced depression by suppressing hippocampal neurogenesis. J Neurochem 2007;103:1843-1854.

20. Oliveira RM, Guimarães FS, Deakin JF. Expression of neuronal nitric oxide synthase in the hippocampal formation in affective disorders. Braz J Med Biol Res 2008;41:333-341.

21. Mistry SJ, Atweh GF. Role of stathmin in the regulation of the mitotic spindle: potential applications in cancer therapy. Mt Sinai J Med 2002; 69:299-304.

22. Shumyatsky GP, Malleret G, Shin RM, Takizawa S, Tully K, Tsvetkov E, et al. stathmin, a gene enriched in the amygdala, controls both learned and innate fear. Cell 2005;123:697-709.

23. López-Figueroa AL, Norton CS, López-Figueroa MO, ArmelliniDodel D, Burke S, Akil H, et al. Serotonin 5-HT1A, 5-HT1B, and 5HT2A receptor mRNA expression in subjects with major depression, bipolar disorder, and schizophrenia. Biol Psychiatry 2004;55:225-233.

24. Kim S, Webster MJ. Postmortem brain tissue for drug discovery in psychiatric research. Schizophr Bull 2009;35:1031-1033. 